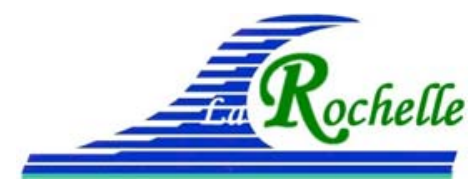

XVèmes Journées Nationales Génie Côtier - Génie Civil

La Rochelle, 29 au 31 mai 2018

DOI:10.5150/jngcgc.2018.086 (C) Editions Paralia CFL

disponible en ligne - http://www.paralia.fr - available online

\title{
Un indice climatique contrôlant les conditions de vagues en hiver le long de la côte atlantique européenne : WEPA (West Europe Pressure Anomaly)
}

\section{Bruno CASTELLE ${ }^{1}$, Guillaume DODET ${ }^{2}$, Gerd MASSELINK $^{3}$ Tim SCOTT $^{3}$ \\ 1. CNRS/Univ. Bordeaux, UMR EPOC, Allée Geoffroy Saint-Hilaire, Pessac, France. bruno.castelle@u-bordeaux.fr}

2. LETG-Brest Geomer UMR 6554 CNRS, Institut Universitaire Européen de la Mer (UBO), Plouzané, France.guillaume.dodet@univ-brest.fr

3. Coastal Processes Research Group, School of Biological and Marine Sciences, Plymouth University, UK.

gerd.masselink@plymouth.ac.uk; timothy.scott@plymouth.ac.uk

\section{Résumé :}

Une méthode statistique s'appuyant sur des simulations rétrospectives de vagues et des champs de pression atmosphérique dans l'Atlantique Nord sur la période 1948-2016 est développée afin de déterminer les gradients de pression optimums permettant d'expliquer la variabilité interannuelle des vagues d'hiver. Les résultats montrent que pour les latitudes supérieures à $52^{\circ} \mathrm{N}$, le gradient optimal est entre l'Islande et le Portugal ou les Açores, ce qui correspond à la définition de l'Oscillation Nord Atlantique (NAO). De la côte ouest irlandaise jusqu'aux côtes marocaines, un autre gradient de pression prend largement le dessus, défini entre l'Irlande et les îles Canaries. Un nouvel indice, appelé West Europe Pressure Anomaly (WEPA) est calculé comme l'anomalie de la différence de pression hivernale (DJFM, décembre à mars) normalisée entre ces deux stations. Dans le Golfe de Gascogne WEPA améliore de 100 à $150 \%$ l'explication de la variabilité interannuelle des hauteurs significatives moyennes et est le seul indice capturant les conditions de l'hiver 2013/2014. Cet hiver a été le plus énergétique depuis au moins 70 ans, et a été caractérisé par une succession de houles de tempête qui ont durablement affecté le littoral atlantique européen. Cet indice ouvre de nouvelles perspectives pour comprendre et prévoir les événements extrêmes sur la façade Atlantique européenne, et des études préliminaires indiquent que cet indice contrôle fortement le débit des fleuves et les températures en Europe de l'Ouest.

\section{Mots-clés :}

Climatologie de vagues, Hivers extrêmes, Variabilité interannuelle, Europe de l'Ouest, Risques côtiers, Simulations rétrospectives, Indices climatiques.

\section{Introduction}

Les conditions de vagues en hiver sont déterminantes pour les aléas d'érosion et de submersion ou encore pour la production d'énergie houlomotrice. Il est reconnu depuis 


\section{Thème 7 - Risques côtiers}

des décennies que les hauteurs moyennes des vagues en hiver présentent une forte variabilité interannuelle, et que cette variabilité exerce sur la plupart des littoraux un fort contrôle sur la variabilité interannuelle du trait de côte (ROBINET et al., 2016) et peut donner lieu à des hivers extrêmes impliquant des érosions massives des littoraux sur plusieurs centaines de kilomètres de linéaire côtier (MASSELINK et al., 2016a). Il devient donc de plus en plus important de lier la variabilité interannuelle de la hauteur des vagues avec les modes de variabilité océaniques et atmosphériques à grande échelle sur des échelles de temps pluri-décennales et au-delà.

En Europe de l'Ouest, l'Oscillation Nord Atlantique (North Atlantic Oscillation, NAO) a pendant longtemps été connue comme le principal mode de variabilité climatique contrôlant les hauteurs des vagues en hiver (BACON \& CARTER, 1993). Toutefois, un certain nombre d'études ont montré que la NAO avait certes un impact important sur le champ de vagues dans les plus hautes latitudes, typiquement au-dessus de $50^{\circ} \mathrm{N}$, mais que cette influence était beaucoup plus subtile plus au sud (DODET et al., 2010). Au sud de $50^{\circ} \mathrm{N}$, d'autres indices climatiques comme les oscillations East Atlantic (EA, le $2^{\text {ème }}$ mode d'oscillation atmosphérique par EOF dans l'Atlantique nord après la NAO) et Scandinavia (SCAND, mode d'oscillation climatique dont le centre est situé sur la péninsule scandinave) exercent souvent un contrôle faible mais par endroits plus important que la NAO sur le champ de vagues (SHIMURA et al., 2013). L'absence de mode de variabilité atmosphérique ou océanique expliquant la plus grande partie de la variabilité du champ de vagues en Europe de l'Ouest est une limitation importante dans l'optique de comprendre et à terme prévoir l'évolution des tendances des hauteurs moyennes d'hiver, de leur variabilité et de leur périodicité, notamment dans un contexte de changement climatique. A titre d'exemple, l'hiver 2013/2014 qui a été de loin l'hiver le plus énergétique depuis au moins 70 ans (MASSELINK et al., 2016a), et qui a causé une érosion massive des littoraux du sud de l'Irlande jusqu'au Maroc (CASTELLE et al., 2015 ; BLAISE et al., 2015 ; MASSELINK et al., 2016b) n'a été capturé par aucun des indices climatiques décrits plus haut. Les indices climatiques peuvent être pourtant d'autant plus pertinents qu'ils arrivent à capturer les hivers extrêmes à l'origine d'aléas majeurs d'érosion et de submersion.

Dans cet article, des simulations rétrospectives de vagues et des champs de pression atmosphérique dans l'Atlantique Nord sur la période 1948-2016 sont utilisées pour développer objectivement un nouvel indice climatique contrôlant l'énergie des vagues en Europe de l'Ouest. L'étude complète est détaillée dans CASTELLE et al. (2017).

\section{Matériel et méthodes}

\subsection{Données atmosphériques et indices climatiques}

Les champs de pression au niveau de la mer PNM (figure 1a) et de vent à 10 m simulés de manière rétrospective toutes les 6 heures sur une grille cartésienne $2,5^{\circ} \times 2,5^{\circ}$ ont été 


\section{XVèmes Journées Nationales Génie Côtier - Génie Civil \\ La Rochelle, 29 au 31 mai 2018}

téléchargés sur le site du National Centers for Environmental Prediction (NCEP) / National Center for Atmospheric Research reanalysis project (KALNAY et al., 1996). Les indices climatiques NAO, EA et SCAND ont été quant à eux obtenus à travers le National Oceanic and Atmospheric Administration (NOAA) Climate Prediction Center (www.cpc.ncep.noaa.gov) par EOF des simulations atmosphériques rétrospectives.

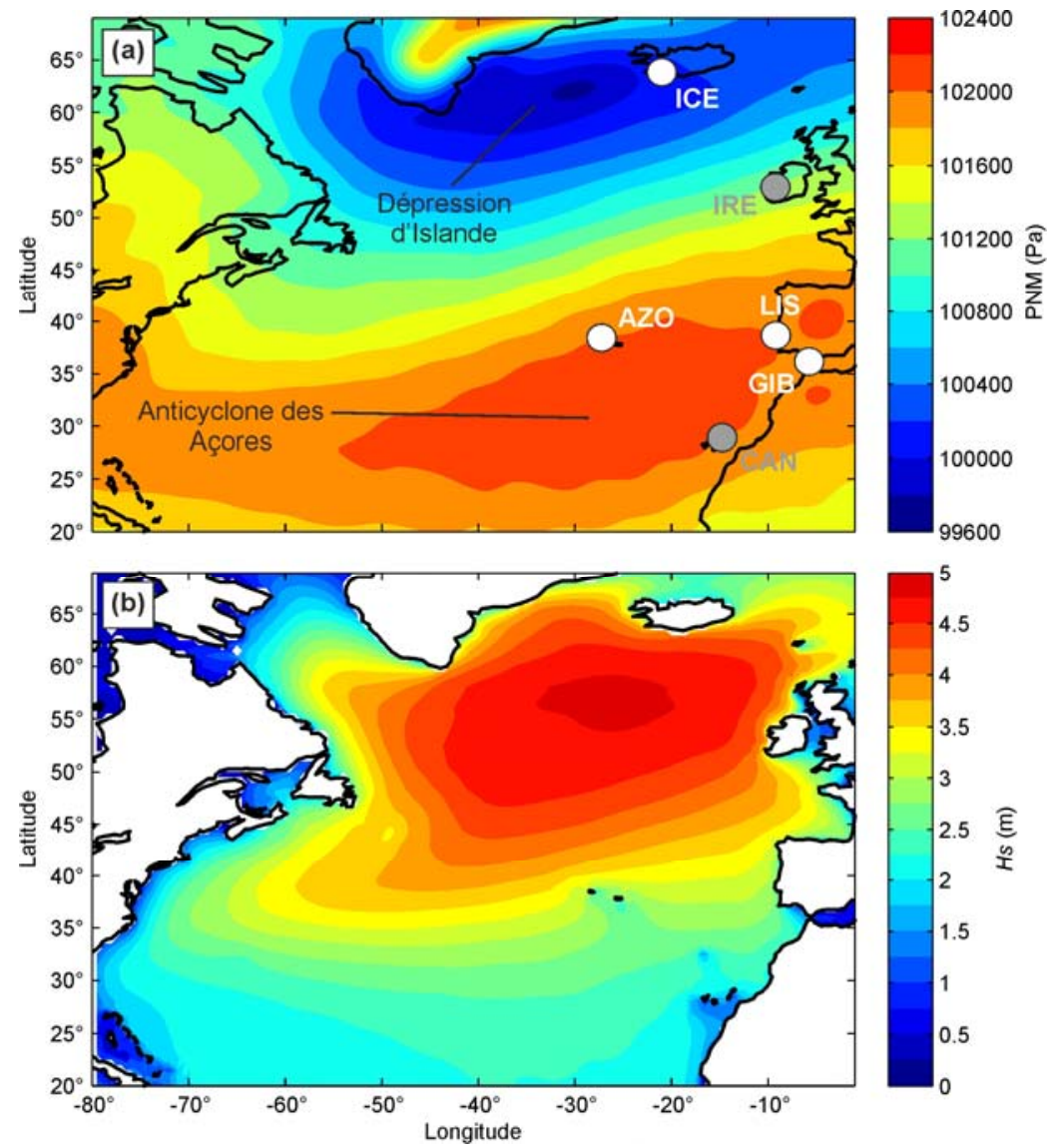

Figure 1. Zoom sur la (a) PNM et (b) Hs moyenne d'hiver (DJFM) sur 1948-2016.

\subsection{Modélisation des vagues}

L'approche détaillée dans MASSELINK et al. (2016a) a été utilisée. Le modèle spectral de vagues WAVEWATCH III V4.18 (TOLMAN, 2014) a été implémenté sur une grille cartésienne de résolution $0,5^{\circ}$ couvrant l'ensemble de l'Atlantique Nord $\left(80^{\circ}-0^{\circ} \mathrm{W} ; 0^{\circ}\right.$ $70^{\circ} \mathrm{N}$ ) forcée par les vents du NCEP afin de simuler l'évolution temporelle de la hauteur significative des vagues $H s$ sur l'ensemble de la période (figure $1 \mathrm{~b}$ ). L'approche a été largement validée à partir d'une dizaine de houlographes disposées tout le long de la côte Ouest européenne (MASSELINK et al., 2016a). 


\section{Thème 7 - Risques côtiers}

\subsection{Détermination des gradients de PNM optimum}

Six bouées virtuelles représentatives de la variabilité spatiale des vagues à l'approche des côtes européenne ont été déterminées (figure $2 b$ ). Les indices climatiques peuvent être calculés par EOF de simulations atmosphériques rétrospectives, ou par le calcul direct de l'anomalie normalisée du gradient de PNM mesurée entre 2 stations météo. Par exemple, la NAO calculée par EOF est corrèle très bien avec l'anomalie normalisée du gradient de PNM mesurée entre une station en Islande une station au niveau de l'anticyclone des Açores comme Lisbonne, aux Açores ou aux Canaries (figure 1a, HURRELL, 1995). Dans cette étude, pour chaque bouée virtuelle, l'anomalie du gradient de PNM moyenne d'hiver corrélant le plus avec la hauteur moyenne des vagues d'hiver est automatiquement recherchée. L'algorithme est appliqué uniquement aux points émergés de la grille afin de pouvoir y rechercher ensuite des séries temporelles in situ de PNM.
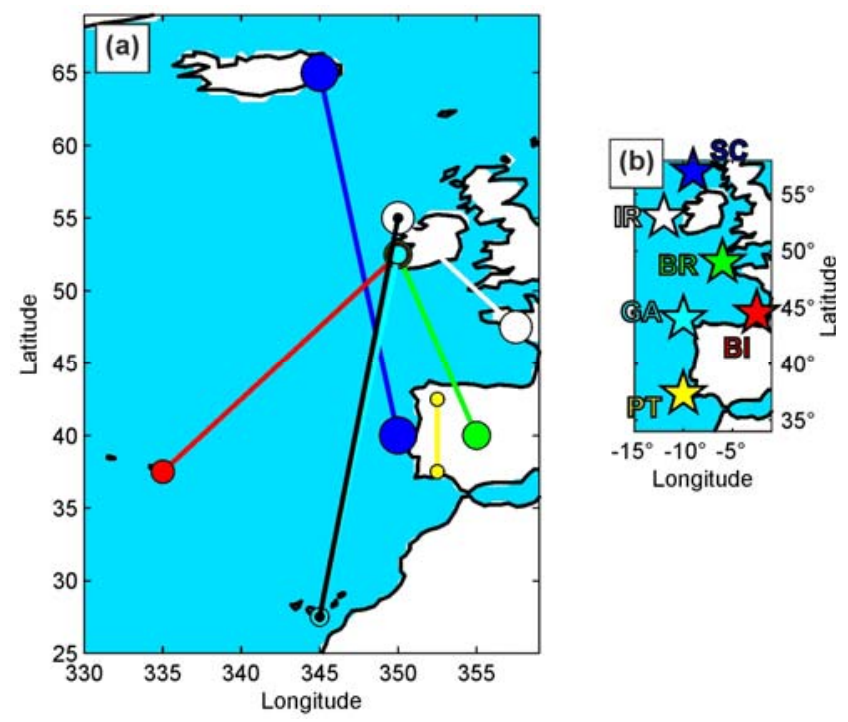

Figure 2. (a) Gradients de PNM optimums expliquant la variabilité des vagues d'hiver aux six bouées virtuelles indiquées dans le panel (b). Dans le panel (a) le gradient optimum noir correspond à la meilleure corrélation en moyenne pour les quatre bouées du Sud BR, BI, GA et PT.

\section{Résultats}

La figure 2a montre que le gradient de PNM optimal pour la bouée la plus au nord se situe entre l'Islande et le sud du Portugal, ce qui correspond à la définition de la NAO (HURRELL, 1995). En descendant vers le sud le long des bouées, le gradient optimal de PNM se déplace également vers le sud, avec des gradients qui ne correspondent à aucun indice climatique connu. Puisqu'il est admis que la NAO exerce un contrôle important sur les bouées du Nord (SC et IR, figure $2 b$ ), le gradient optimal en moyenne pour les quatre bouées du Sud (BR, BI, GA et PT, figure 2b) est recherché, et 


\section{XVèmes Journées Nationales Génie Côtier - Génie Civil \\ La Rochelle, 29 au 31 mai 2018}

l'algorithme détermine qu'il s'agit du gradient de PNM entre l'Irlande et les îles Canaries. Notre nouvel indice climatique, appelé West Europe Pressure Anomaly (WEPA) est alors calculé comme la différence PNM hivernale (DJFM) normalisée entre la station Valentia (Irlande) et Santa Cruz de Tenerife (Canaries) où des mesures in situ long terme ont pu être collectées.

La figure 3 montre la distribution spatiale du coefficient de corrélation $R$ entre les moyennes hivernale de $H s$ et les différents indices climatiques. En accord avec les études précédentes, la NAO est très bien corrélée avec les hauteurs significatives audessus de $52^{\circ} \mathrm{N}$, mais $R$ décroit rapidement vers le sud (figure 3a). SCAND donne des corrélations faibles dans tout le domaine alors que EA donne des résultats meilleurs que la NAO au sud de $52^{\circ} \mathrm{N}$, même si avec $R<0,65$ EA explique systématiquement moins de $42 \%$ de la variabilité interannuelle observée (figure 3c). Par contre WEPA obtient systématiquement $R>0,7$ de la côte sud irlandaise jusqu'aux côtes marocaines, et explique donc systématiquement plus de $50 \%$ de la variabilité interannuelle observée. La figure 4 montre l'augmentation de l'explication de la variabilité interannuelle des vagues d'hiver avec WEPA comparée au meilleur indice localement entre NAO, SCAND et EA. Les résultats montrent qu'au sud de $52^{\circ} \mathrm{N}$ WEPA améliore systématiquement l'explication des variabilités interannuelles des hauteurs moyennes de vagues, voire même systématiquement entre 100 à 150\% dans le Golfe de Gascogne (figure 4a). Cette amélioration devient encore plus importante lorsque les événements extrêmes sont considérés (e.g. le quantile $99,5 \%$ des $H s$ dans la figure $4 b$ ).
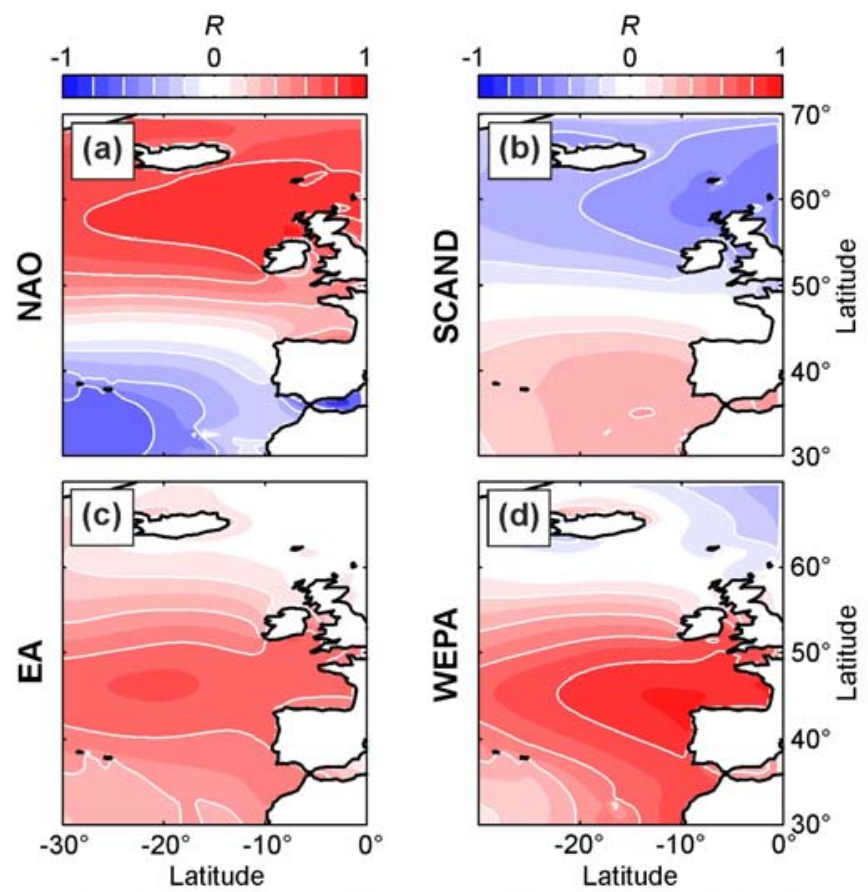

Figure 3. Distribution spatiale du coefficient de corrélation $R$ entre les moyennes hivernale de Hs et les indices climatiques NAO, SCAND EA et WEPA. 

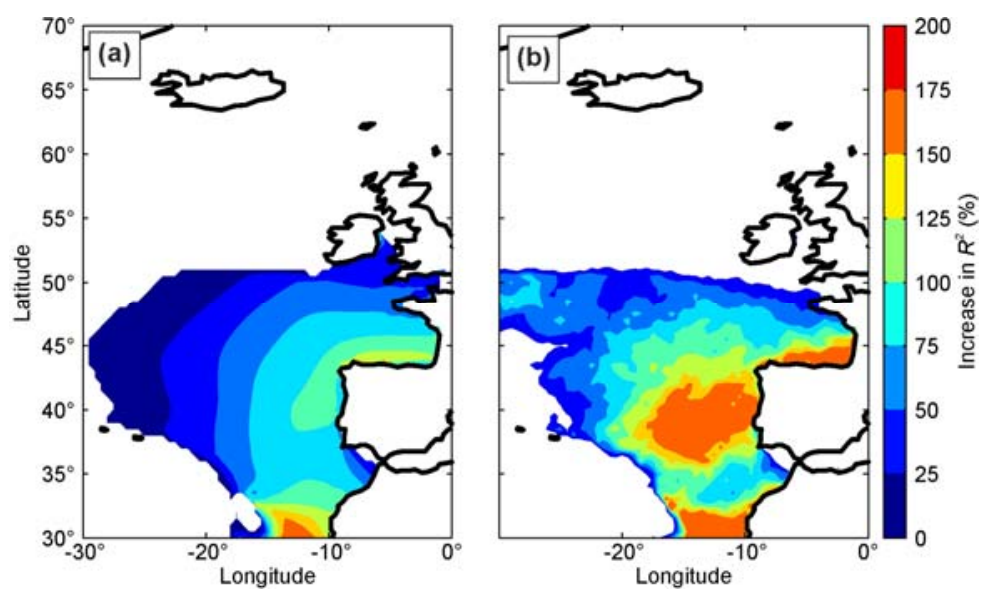

Figure 4. Amélioration en \% de l'explication de la variabilité interannuelle des vagues avec WEPA comparée au meilleur localement des trois autres indices climatiques (NAO, SCAND et EA) pour (a) Hs et (b) Hs, 99.5\%.
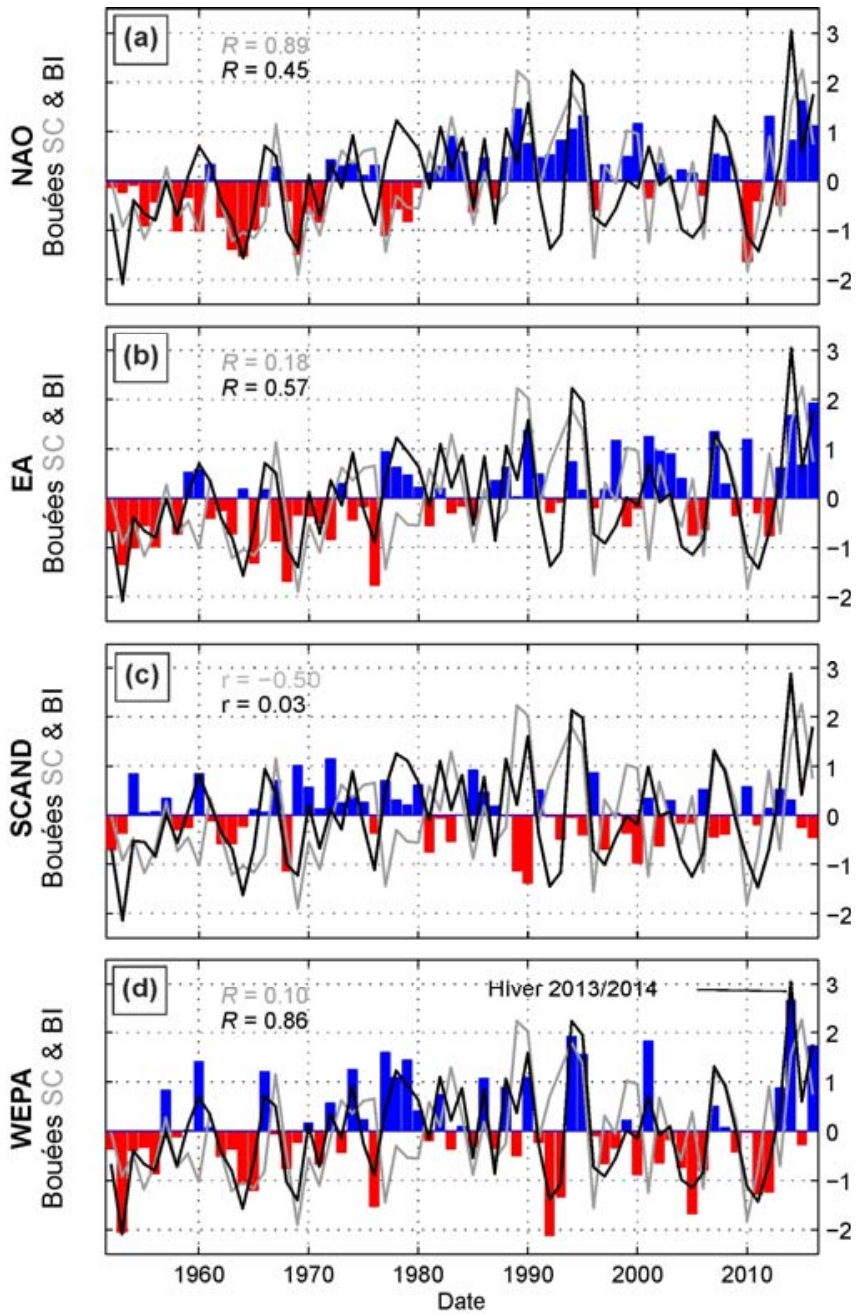

Figure 5. Série temporelle de la hauteur hivernale des vagues normalisée aux bouées $S C$ et BI, et des indices climatiques (barres colorées) NAO, SCAND, EA et WEPA. 


\section{XVèmes Journées Nationales Génie Côtier - Génie Civil \\ La Rochelle, 29 au 31 mai 2018}

La figure 5 montre les séries temporelles des indices climatiques et de la hauteur des vagues d'hiver normalisée aux bouées SC et BI. Avec un coefficient de corrélation $R=$ 0,89 avec les hauteurs à la bouée $\mathrm{SC}$, la NAO exerce un fort contrôle sur les vagues le long des côtes écossaise, et est même l'indice optimale comme démontré par les résultats de notre algorithme (figure 5a). Par contre, plus au sud à BI, la NAO exerce un contrôle relativement faible $(R=0,45$, figure 5a) expliquant donc que $20 \%$ de la variabilité interannuelle des vagues d'hiver. Les indices EA (figure 5b) et SCAND (figure 5c) exerce un contrôle beaucoup plus faibles sur les vagues d'hiver à SC et BI, avec toutefois EA expliquant près de $32 \%$ de la variabilité observées à BI. WEPA quant à lui est très peu corrélé aux hauteurs de vagues d'hiver à $\mathrm{SC}(R=0,10$, figure $5 \mathrm{~d})$. Par contre à BI, WEPA obtient $R=0,86$ et explique donc $74 \%$ de la variabilité observée dans le Golfe de Gascogne, avec une explication maximisée au large de la Galice (85\%). En outre, WEPA et le seul indice qui capture l'hiver 2013/2014 (figure 5d) qui a été de loin de le plus énergétique de la côte irlandaise jusqu'aux côtes marocaines depuis au moins 1948 (MASSELINK et al., 2016a). En effet l'hiver 2013/2014 correspond au plus haut WEPA de la série $(+2,65)$, alors que le $2^{\text {ème }}$ WEPA le plus élevé a été mesuré pendant l'hiver 1993/1994 (+1,92).

\section{Conclusions}

Un nouvel indice climatique est proposé qui permet d'expliquer la variabilité interannuelle des vagues d'hiver en Europe de l'Ouest Le West Europe Pressure Anomaly (WEPA) est défini comme l'anomalie de la différence de pression normalisée mesurée entre les stations Valentia (Irlande) et Santa Cruz de Tenerife (Canaries). La phase positive de WEPA reflète une intensification et une translation vers le sud du gradient de pression entre la dépression islandaise et l'anticyclone des Açores (voir CASTELLE et al., 2017). WEPA surpasse les autres indices climatiques sur les côtes européennes et marocaines au sud de $52^{\circ} \mathrm{N}$ et améliore de 100 à $150 \%$ 1'explication de la variabilité interannuelle des hauteurs moyennes dans le Golfe de Gascogne. C'est également le seul indice climatique capturant l'hiver 2013/2014, hiver le plus énergétique depuis au moins 70 ans qui a durablement affecté le littoral atlantique européen.

\section{Références bibliographiques}

BACON S., CARTER D.J.T. (1993). A connection between mean wave height and atmospheric pressure gradient in the North Atlantic. Int. J. Climatol., Vol. 13, pp 423436. https://doi.org/10.1002/joc.3370130406

BLAISE E., SUANEZ S., STEPHAN P., FICHAUT F., DAVID L., CUQ V., AUTRET R., HOURON J., ROUAN M., FLOC'H F., ARDHUIN F., CANCOUËT R., DAVIDSON R., COSTA S., DELACOURT C. (2015). Bilan des tempêtes de l'hiver 


\section{Thème 7 - Risques côtiers}

2013-2014 sur la dynamique de recul du trait de côte en Bretagne. Géomorphologie : Relief, Processus, Environnement, Vol. 21, pp 267-292. https://doi.org/10.4000/geomorphologie.11104 CASTELlE B., MARIEU V., BUJAN S., SPLINTER K.D., ROBINET A., SENECHAL N., FERREIRA S. (2015). Impact of the winter 2013-2014 series of severe Western Europe storms on a double-barred sandy coast: Beach and dune erosion and megacusp embayments. Geomorphology, Vol. 238, pp 135-148. https://doi.org/10.1016/j.geomorph.2015.03.006

CASTELLE B., DODET G., MASSELINK G., SCOTT T. (2017). A new climate index controlling winter wave activity along the Atlantic coast of Europe: The West Europe Pressure Anomaly. Geophys. Res. Lett., Vol. 44, 9p. https://doi.org/10.1002/2016GL072379

DODET G., BERTIN X., TABORDA R. (2010). Wave climate variability in the NorthEast Atlantic Ocean over the last six decades. Ocean Modell., Vol. 31, pp 120-131. https://doi.org/10.1016/j.ocemod.2009.10.010

HURRELL J.W. (1995). Decadal trends in the North Atlantic Oscillation: Regional temperatures and precipitation. Science, Vol. 269, pp 676-679, https://doi.org/10.1126/science.269.5224.676

KALNAY E., KANAMITSU M., KISTLER R., et al. (1996). The NCEP/NCAR 40-year reanalysis project, Bull. Am. Meteorol. Soc., Vol. 77, pp 437-471. https://doi.org/10.1175/1520-0477(1996)077<0437:TNYRP>2.0.CO;2

MASSELINK G., CASTELlE B., SCOTT T., DODET G., SUANEZ S., JACKSON D., FLOC'H F. (2016a). Extreme wave activity during 2013/2014 winter and morphological impacts along the Atlantic coast of Europe. Geophys. Res. Lett., Vol. 43, pp 2135-2143. https://doi.org/10.1002/2015GL067492

MASSELINK G., SCOTT T., POATE T., RUSSELL P., DAVIDSON M., CONLEY D. (2016b). The extreme 2013/2014 winter storms: Hydrodynamic forcing and coastal response along the southwest coast of England. Earth Surf. Processes Landforms, Vol. 41, pp 378-391. https://doi.org/10.1002/esp.3836

ROBINET A., CASTELlE B., IDIER D., LE COZANNET G., DEQUE M., CHARLES E. (2016). Statistical modeling of interannual shoreline change driven by North Atlantic climate variability spanning 2000-2014 in the Bay of Biscay. Geo-Mar. Lett., Vol. 36, pp 479-490. https://doi.org/10.1007/s00367-016-0460-8

SHIMURA T., MORI N., MASE H. (2013). Ocean Waves and Teleconnection Patterns in the Northern Hemisphere. J. Climate, Vol. 26, pp 8654-8670. https://doi.org/10.1175/JCLID-12-00397.1

TOLMAN H.L. (2014). User manual and system documentation of WAVEWATCH III version 4.18, NOAA/NWS/NCEP/ MMAB Tech. Note 316, 194 pp, NOAA/NWS/NCEP/MMAB, College Park, Md. URL : http://tel.archives-ouvertes.fr/tel-00380212 